\title{
Chinese SLE Treatment and Research Group Registry: III. Association of Autoantibodies with Clinical Manifestations in Chinese Patients with Systemic Lupus Erythematosus
}

\author{
Jing Li, ${ }^{1}$ Xiaomei Leng, ${ }^{1}$ Zhijun Li, ${ }^{2}$ Zhizhong Ye, ${ }^{3}$ Caifeng Li, ${ }^{4}$ Xiaofeng Li, ${ }^{5}$ Ping Zhu, \\ Zhengang Wang, ${ }^{7}$ Yi Zheng, ${ }^{8}$ Xiangpei Li, ${ }^{9}$ Miaojia Zhang, ${ }^{10}$ Xin-Ping Tian, ${ }^{1}$ Mengtao Li, ${ }^{1}$ \\ Jiuliang Zhao, ${ }^{1}$ Feng-Chun Zhang, ${ }^{1}$ Yan Zhao, ${ }^{1}$ and Xiaofeng Zeng ${ }^{1,11}$ \\ ${ }^{1}$ Key Laboratory of Rheumatology and Clinical Immunology, Department of Rheumatology, Peking Union Medical College Hospital, \\ Ministry of Education, No. 1 Shuaifuyuan, Beijing 100730, China \\ ${ }^{2}$ Department of Rheumatology, The Affiliated Hospital of Bengbu Medical College, No. 2600 Do Hai Da Dao, \\ Anhui 233030, China \\ ${ }^{3}$ Department of Rheumatology, The Fourth People's Hospital of Shenzhen Affiliated to Guangdong Medical College, \\ No. 22 Nong Lin Lu, Guangdong 518034, China \\ ${ }^{4}$ Department of Rheumatology, Beijing Children's Hospital Affiliated to Capital Medical University, No. 56 Nan Li Shi Lu, \\ Beijing 100045, China \\ ${ }^{5}$ Department of Rheumatology, The Second Hospital of Shanxi Medical University, No. 382 Wu Yi Lu, \\ Shanxi 030001, China \\ ${ }^{6}$ Department of Clinical Immunology and Rheumatology, Xijing Hospital Affiliated to the Fourth Military Medical University, \\ No. 15 Chang Le Xi Lu, Shanxi 710032, China \\ ${ }^{7}$ Department of Rheumatology, Beijing Tongren Hospital Affiliated to Capital Medical University, No. 1 Dong Jiao Min Xiang, \\ Beijing 100730, China \\ ${ }^{8}$ Department of Rheumatology, Beijing Chao-yang Hospital Affiliated to Capital Medical University, No. 8 Gong Ti Nan Lu, \\ Beijing 100020, China \\ ${ }^{9}$ Department of Rheumatology, Anhui Provincial Hospital, No. 17 Lu Jiang Lu, Anhui 230001, China \\ ${ }^{10}$ Department of Rheumatology, Jiangsu Provincial People's Hospital, No. 30 Guang Zhou Lu, Jiangsu 210029, China \\ ${ }^{11}$ Department of Rheumatology, Peking Union Medical College and Chinese Academy of Medical Sciences, \\ Peking Union Medical College Hospital, Beijing 100730, China
}

Correspondence should be addressed to Xiaofeng Zeng; zengxfpumc@gmail.com

Received 11 January 2014; Revised 27 March 2014; Accepted 27 March 2014; Published 23 April 2014

Academic Editor: Marvin J. Fritzler

Copyright (C) 2014 Jing Li et al. This is an open access article distributed under the Creative Commons Attribution License, which permits unrestricted use, distribution, and reproduction in any medium, provided the original work is properly cited.

We investigated the characteristics of Chinese SLE patients by analyzing the association between specific autoantibodies and clinical manifestations of 2104 SLE patients from registry data of CSTAR cohort. Significant $(P<0.05)$ associations were found between anti-Sm antibody, anti-rRNP antibody, and malar rash; between anti-RNP antibody, anti-SSA antibody, and pulmonary arterial hypertension (PAH); between anti-SSB antibody and hematologic involvement; and between anti-dsDNA antibody and nephropathy. APL antibody was associated with hematologic involvement, interstitial lung disease, and a lower prevalence of oral ulcerations $(P<0.05)$. Associations were also found between anti-dsDNA antibody and a lower prevalence of photosensitivity, and between anti-SSA antibody and a lower prevalence of nephropathy $(P<0.05)$. Most of these findings were consistent with other studies in the literature but this study is the first report on the association between anti-SSA and a lower prevalence of nephropathy. The correlations of specific autoantibodies and clinical manifestations could provide clues for physicians to predict organ damages in SLE patients. We suggest that a thorough screening of autoantibodies should be carried out when the diagnosis of SLE is established, and repeated echocardiography annually in SLE patients with anti-RNP or anti-SSA antibody should be performed. 


\section{Introduction}

Systemic lupus erythematosus (SLE) is one of the most complicated autoimmune diseases. It could involve almost all organs or systems and presents with protean clinical manifestations [1]. In general, SLE can be divided into several subgroups based on specific clinical features including age, gender, and autoantibodies pattern, and the prognosis of different subgroups varies [2]. Anti-Sm antibody is considered as the marker autoantibody for the diagnosis of SLE with reported positivity ranged from $15.4 \%$ to $21.8 \%$ [3, 4]. Antidouble stranded DNA (anti-dsDNA) antibody is another specific autoantibody for SLE and has been proven to be associated with disease activity of SLE [5]. In order to understand SLE better, the association between clinical features of SLE and other anti-extractable nuclear antigen (ENA) antibodies (e.g., anti-SSA, anti-SSB, anti-RNP, and anti-rRNP antibodies) had been investigated by many research groups $[6,7]$. In our study, we analyzed the associations between clinical manifestations and autoantibody patterns in Chinese SLE patients based on the data from Chinese SLE Treatment and Research group (CSTAR) registry. CSTAR is the first online registry of Chinese SLE patients and is supported by the Chinese National Key Technology R\&D Program. This registry has depicted major clinical characteristics of lupus in Chinese patients [8].

\section{Methods and Patients}

2.1. Patients. CSTAR launched the first registry project of Chinese SLE patients in 2009, which was approved by the Institute Review Board (IRB) of Peking Union Medical College Hospital (PUMCH). Other centers had received ethical approval by the local IRB. All investigators were trained for the diagnosis, history review, disease activity evaluation, laboratory examinations, data input, and sample collection by local or nationwide training programs. This ongoing registry had recruited 2170 Chinese SLE patients who fulfilled the SLE classification criteria revised by the American College of Rheumatology (ACR) in 1997 [9] during the period between April 2009 and February 2010. Patients were required to fulfill at least 4 of the following 11 criteria: (1) malar rash; (2) discoid rash; (3) photosensitivity; (4) oral or nasopharyngeal ulceration; (5) nonerosive arthritis involving 2 or more peripheral joints; (6) pleuritis or pericarditis; (7) nephropathy: persistent proteinuria $>0.5$ grams per day or cellular casts; (8) neurologic involvement: seizures or psychosis in the absence of offending drugs or known metabolic derangements; (9) hematologic involvement: hemolytic anemia with reticulocytosis or leukopenia $\left(<4,000 / \mathrm{mm}^{3}\right.$ on $\geq 2$ occasions) or lymphopenia $\left(<1,500 / \mathrm{mm}^{3}\right.$ on $\geq 2$ occasions $)$ or thrombocytopenia $\left(<100,000 / \mathrm{mm}^{3}\right)$ in the absence of offending drugs; (10) immunologic disorder: antibody to native doublestranded DNA in abnormal titer or presence of antibody to Sm nuclear antigen or positive finding of antiphospholipid antibodies; (11) positive antinuclear antibody. We confirmed positive finding of antiphospholipid antibodies by an abnormal serum level of IgG or IgM anticardiolipin antibodies, an abnormal serum level of anti- $\beta 2$ glucoprotein I, or a positive test result for lupus anticoagulant. In this study, we analyzed baseline data of 2104 patients (including 190 male and 1914 female patients) (Table 1$)$.

2.2. Methods. Each center of the CSTAR has provided uniform evaluations and recorded data following the same protocol and operational procedures. Clinical manifestations and systemic involvement of SLE patients were collected, evaluated, and the relevant data were entered into the online CSTAR registry database. Pulmonary arterial hypertension (PAH) was defined as a resting systolic PAP (PASP) $\geq 40 \mathrm{mmHg}$ estimated by echocardiography [10] without chronic lung conditions, cardiac valvular, or cardiomyopathic complications. Interstitial lung disease (ILD) was detected by chest X-ray or computerized tomography without infective infiltrations. Patients who had ILD with known causes or PAH were excluded when the association between autoantibodies and ILD in this study was analyzed.

The autoantibodies were measured at the local labs of each center, including anti-nuclear antibody (ANA), antidouble stranded DNA (anti-dsDNA) antibody, anti-Sm antibody, anti-ribosomal RNA-protein (anti-rRNP) antibody, anti-SSA antibody, anti-SSB antibody, anti-ul small-nuclear RNA-protein (anti-RNP) antibody, and anti-phospholipid (APL) antibody. Most centers detected ANA and anti-dsDNA antibody using immunofluorescence assay with Hep-2 cell line and anti-extractable nuclear antigen (ENA) antibody (including anti-Sm, anti-SSA, anti-SSB, anti-RNP, and antirRNP antibodies) was tested with immunoblotting assay. The APL antibody was tested using enzyme-linked immunosorbent assay (anticardiolipin and anti- $\beta 2$ glucoprotein I antibody) or dilute Russell viper venom test (lupus anticoagulant) when anti-phospholipid syndrome was suspected but these tests were not mandatory.

2.3. Statistical Analysis. Statistical analysis was performed by using SPSS 17.0 for WINDOWS. Positivity of autoantibodies in SLE patients with different clinical manifestations was expressed as patient number with percentage (\%) in brackets (Table 4). Chi-square tests were performed to detect the associations between clinical manifestations and autoantibody patterns. Since there was no expected frequency $<5$, Fisher's exact test was not used. Cluster analysis with Ward's method was performed to investigate the relationship between autoantibodies. All tests of significance were twosided and a $P$ value of $<0.05$ was considered to be statistically significant.

\section{Results}

The autoantibody profile of this study included the presence of ANA in 2063 (98.1\%), anti-dsDNA antibody in 699 (33.2\%), anti-Sm antibody in 350 (16.6\%), anti-RNP antibody in 189 (8.9\%), anti-SSA antibody in 497 (23.6\%), anti-SSB antibody in 224 (10.7\%), and anti-rRNP antibody in 255 $(12.7 \%)$ cases. APL antibody was tested in 937 patients with a positivity of $44.1 \%$ (414/937). 199 patients (9.5\%) were 
TABLE 1: The baseline characteristics of 2104 SLE patients from CSTAR cohort study.

\begin{tabular}{lcc}
\hline & & $\%$ \\
\hline Female & 1914 & 91.0 \\
Male & 190 & 9.0 \\
Age at onset (years) & $29.2 \pm 12.1$ (range 1.4 68.9) & \\
Age at diagnosis (years) & $30.3 \pm 12.3$ (range 4 77) & \\
Age at entry (years) & $32.7 \pm 12.7$ (range 5 78) & \\
Disease duration (months) & $41.9 \pm 58.8$ (range 1 468) \\
SLE disease activity index & & \\
at entry & & \\
$\quad 0 \sim 4$ & 532 & 25.3 \\
$5 \sim 9$ & 587 & 28.9 \\
$10 \sim 14$ & 591 & 18.7 \\
$>14$ & 394 & \\
\hline
\end{tabular}

demonstrated to have both anti-SSA antibody and anti-SSB antibody and 142 patients (6.7\%) have anti-Sm antibody and anti-RNP antibody, simultaneously (Table 2).

Clinical manifestations found in our study included malar rash in 1009 (47.9\%), discoid skin lesions in 118 (5.6\%), photosensitivity in $526(25.0 \%)$, oral ulcer in $466(22.1 \%)$, arthritis in 1147 (54.5\%), serositis in 345 (16.4\%), nephropathy in 988 (47.4\%), hematological involvement, including leukocytopenia, hemolytic anemia, and thrombocytopenia, in 1181 (56.1\%), and neurological involvement (neuropsychological lupus) in 101 (4.8\%) patients. The prevalence of ILD and PAH was $4.2 \%(86 / 2024)$ and $3.8 \%(74 / 1934)$, respectively in this registry database (Table 3 ).

The association analysis between clinical manifestations and autoantibodies revealed that there were associations between anti-Sm antibody $(P<0.001)$, anti-rRNP antibody $(P<0.05)$, and malar rash; between anti-dsDNA antibody and nephropathy; between anti-RNP antibody, anti-SSA antibody and pulmonary arterial hypertension (PAH); and between anti-SSB antibody and hematological involvement $(P<0.05)$. Significant associations were also found between anti-dsDNA antibody and a lower prevalence of photosensitivity and between anti-SSA antibody and a lower prevalence of nephropathy $(P<0.05)$. APL antibody was associated with hematologic involvement, interstitial lung disease (ILD), and a lower prevalence of oral ulcerations $(P<0.05)$ (Table 4$)$.

Using cluster analysis, we identified five clusters of antibodies. Cluster 1 consisted of antibodies to Sm and RNP and cluster 2 consisted of antibodies to SSA and SSB. Clusters 3, 4 , and 5 consisted of antibodies to ribosomal P, dsDNA, and APL, respectively (Figure 1).

\section{Discussion}

Diffuse connective tissue diseases (CTDs) are characterized by the presentation of specific profiles of autoantibodies, and SLE is the prototype of diffuse CTDs, which could involve almost all systems [1]. The typical pathological feature of SLE is systemic vasculitis with immune complex deposition [11].
TABLE 2: The profile of autoantibodies in 2104 SLE patients from CSTAR cohort study.

\begin{tabular}{lcc}
\hline & $\begin{array}{c}\text { Patients } \\
\text { number }\end{array}$ & $\begin{array}{c}\text { Positivity } \\
(\%)\end{array}$ \\
\hline $\begin{array}{l}\text { Anti-nuclear antibody (ANA) } \\
\text { Anti-double stranded DNA } \\
\text { (anti-dsDNA) antibody }\end{array}$ & 2063 & 98.1 \\
Anti-Sm antibody & 699 & 33.2 \\
Anti-SSA antibody & 350 & 16.6 \\
$\begin{array}{l}\text { Anti-SSB antibody } \\
\text { Anti-ul small-nuclear RNA-protein } \\
\text { (anti-RNP) antibody }\end{array}$ & 497 & 23.6 \\
$\begin{array}{l}\text { Anti-ribosomal RNA-protein } \\
\text { (anti-rRNP) antibody }\end{array}$ & 224 & 10.7 \\
$\begin{array}{l}\text { Anti-phospholipid (APL) antibody } \\
\text { Anti-SSA and anti-SSB antibody } \\
\text { positive simultaneously }\end{array}$ & $414 / 937^{\$}$ & 8.9 \\
$\begin{array}{l}\text { Anti-Sm and anti-RNP antibody } \\
\text { positive simultaneously }\end{array}$ & 199 & 12.7 \\
\$ctually detected number of patients. & 142 & 9.5 \\
Ant & &
\end{tabular}

TABLE 3: The profile of clinical manifestations in 2104 SLE patients from CSTAR cohort study.

\begin{tabular}{lcc}
\hline & $\begin{array}{c}\text { Patients } \\
\text { number }\end{array}$ & $\begin{array}{c}\text { Positivity } \\
(\%)\end{array}$ \\
\hline Malar rash & 1009 & 47.9 \\
Discoid lesions & 118 & 5.6 \\
Photosensitivity & 526 & 25.0 \\
Oral ulcerations & 466 & 22.1 \\
Arthritis & 1147 & 54.5 \\
Serositis & 345 & 16.4 \\
Nephropathy & 998 & 47.4 \\
Hematological involvement & 1181 & 56.1 \\
(hematocytopenia) & 101 & 4.8 \\
Neurological involvement & $86 / 2024^{\$}$ & 4.2 \\
Interstitial lung disease & $74 / 1934^{\$}$ & 3.8 \\
Pulmonary arterial hypertension & &
\end{tabular}

$\$$ Actually detected number of patients.

It is rational to propose that some autoantibodies may be associated with specific clinical features of SLE.

Anti-dsDNA has been proven to be a pathogenic autoantibody in SLE and has been reported to be associated with renal damage, leukopenia, anemia, and urine cellular casts in SLE patients $[6,7,12]$. Chien et al. discovered that there were associations between anti-dsDNA antibodies and multiple clinical manifestations of SLE patients, such as Raynaud's phenomenon, photosensitivity, arthritis, hypocomplementemia, thrombocytopenia, proteinuria, and serositis, but with a small number of patients ( 80 patients) [13]. Our study confirmed that anti-dsDNA was associated with nephropathy (Table 4) which was also reported by 
Lu et al. and Alba et al. $[12,14]$. The association between antidsDNA antibody and a lower prevalence of photosensitivity observed in our study was contradictory to Smikle et al. [4]. The difference may be due to different ethnical background and sample size.

As a marker autoantibody of SLE, anti-Sm antibody was found to be associated with malar rash, discoid rash, pericarditis, and leukopenia in studies by Tang et al. and Lu et al. $[7,12]$ However, the pathogenic characteristics of anti-Sm antibody were controversial $[6,12,14,15]$. Our study also revealed the association of anti-Sm antibody with malar rash, and malar rash is a characteristic skin lesion of SLE patients. But the association between anti-Sm antibody and other organ damage could not be detected in our study (Table 4).

Anti-RNP antibody was thought to be related to Raynaud's phenomenon and PAH by many physicians. The association between anti-RNP and Raynaud's phenomenon was confirmed by Hoffman et al. and Tang et al. [6, 7]. Both Raynaud's phenomenon and anti-RNP antibody are considered as risk factors for PAH associated with CTDs and represent the presence of vasculopathy [16]. Anti-RNP antibody was also found to be associated with photosensitivity [7], lymphopenia [12], and leukopenia [6]. In our study, the Raynaud's phenomenon was not included in the clinical manifestations analysis, but the association between antiRNP antibody and PAH in patients with SLE was discovered (Table 4).

Anti-SSA and anti-SSB antibody are frequently found in SLE patients with the positivity ranged from $34 \%$ to $83 \%$ in different reports $[8,17]$ and higher prevalence of anti-SSA/SSB antibody could be observed in SLE patients associated with secondary Sjogren's syndrome [18]. Anti-SSA antibody was found to be associated with neonatal heart block [19], xerophthalmia/xerostomia, and photosensitivity [20]. Anti-SSB body was found to be associated with hematological disorder, proteinuria, malar rash [12], and pericarditis [6]. In this study, we found the associations between anti-SSA antibody and PAH, between anti-SSB antibody and hematological involvement, and between anti-SSA antibody and a lower prevalence of nephropathy (Table 4). This study is the first report that anti-SSA antibody might be a predictor of PAH in SLE patients according to our knowledge. Since we have known that anti-SSA antibody is one of the diagnostic criteria for Sjogren's syndrome [21] and PAH is also a rare manifestation of patients with primary Sjogren's syndrome [22], we propose that more attention should be paid to screen for PAH in SLE patients with anti-SSA antibody. But further studies are needed to clarify this association in the future. The association between anti-SSA antibody and a lower prevalence of lupus nephritis was reported by Chien and coresearchers in a small sample size study [13] and Tapanes et al. found that anti-SSA antibody may correlate with favorable prognosis of lupus nephritis [23]. But Vila et al. found the opposite results in 201 Puerto Ricans patients with SLE [24]. We confirmed the association between anti-SSA antibody and a lower prevalence of nephropathy in Chinese SLE patients and this association may suggest a protective role of anti-SSA antibody in lupus nephritis.
The hematological disorder in SLE patients with positive anti-SSB antibody was primarily thrombocytopenia in our study and the association between anti-SSB antibody and thrombocytopenia was reported by Unal et al. in a case report [25]. Large sample studies are needed to clarify the real association between the hematological disorders and antiSSB antibody.

Anti-rRNP antibody is regarded as a specific autoantibody of SLE [26] and it is thought to be associated with neuropsychological manifestations of SLE patients [27]. This relationship was not proven in our study, what may be due to the small amount of patients with neuropsychological manifestations in our registry. The association of anti-rRNP antibody and malar rash was found in our study (Table 4), which was consistent with anti-Sm antibody.

The APL antibody was tested in 937 patients when antiphospholipid syndrome was suspected in our cohort study. The association between APL antibody and hematological involvement (mainly thrombocytopenia) was deductible since phospholipid is an innate component of blood cells. McClain and coresearchers have found that APL antibody appeared prior to diagnosis of SLE and this group of autoantibodies are associated with many SLE features, including malar rash, discoid lesions, photosensitivity, renal disorder, neurological disorder, hemolytic anemia, and thrombocytopenia [28]. Saches and coresearchers have demonstrated that APL antibody is associated with spontaneous abortion, thrombocytopenia, livedo reticularis, and a positive direct Coombs' test in SLE patients [29]. The association between APL antibody and ILD found in our study may be related to the microvessel injuries resulted from microemboli or immune-complex deposition (Table 4). Kanakis et al. have reviewed the pulmonary manifestations in patients with antiphospholipid syndrome including fibrosing alveolitis [30]. We confirmed the association between antiphospholipid antibody and ILD, which is a rare feature of SLE. Neurological involvement in SLE patients is thought to be correlated with APL antibody [31, 32], but previous studies have not shown this association $[28,29]$, perhaps due to the low incidence of neuropsychological lupus in SLE patients. We demonstrated a tendency $(P=0.061)$ of association between APL antibody and neurological involvement in our study (Table 4). Further studies with more patients are needed.

Using cluster analysis, we identified five clusters of autoantibodies. Antibodies to Sm and RNP clustered together early. Cluster 2 consisted of antibodies to SSA and SSB. The other clusters consisted of individual antibodies to ribosomal $\mathrm{P}$ (rRNP), dsDNA, and APL, respectively (Figure 1). Our result is in accordance with previous studies $[6,33]$.

We summarize the associations with statistical significance between specific autoantibodies and clinical manifestations revealed by different study groups in Table 5. Most of our findings are consistent with studies in the literature, but the associations between anti-RNP antibody and PAH; between anti-SSA and PAH; and between APL antibody and ILD were first discovered by our study. We always repeated echocardiography if $\mathrm{PAH}$ was suspected in the first echocardiogram examination. Right heart catheter (RHC) is not feasible for general screening and repeated 


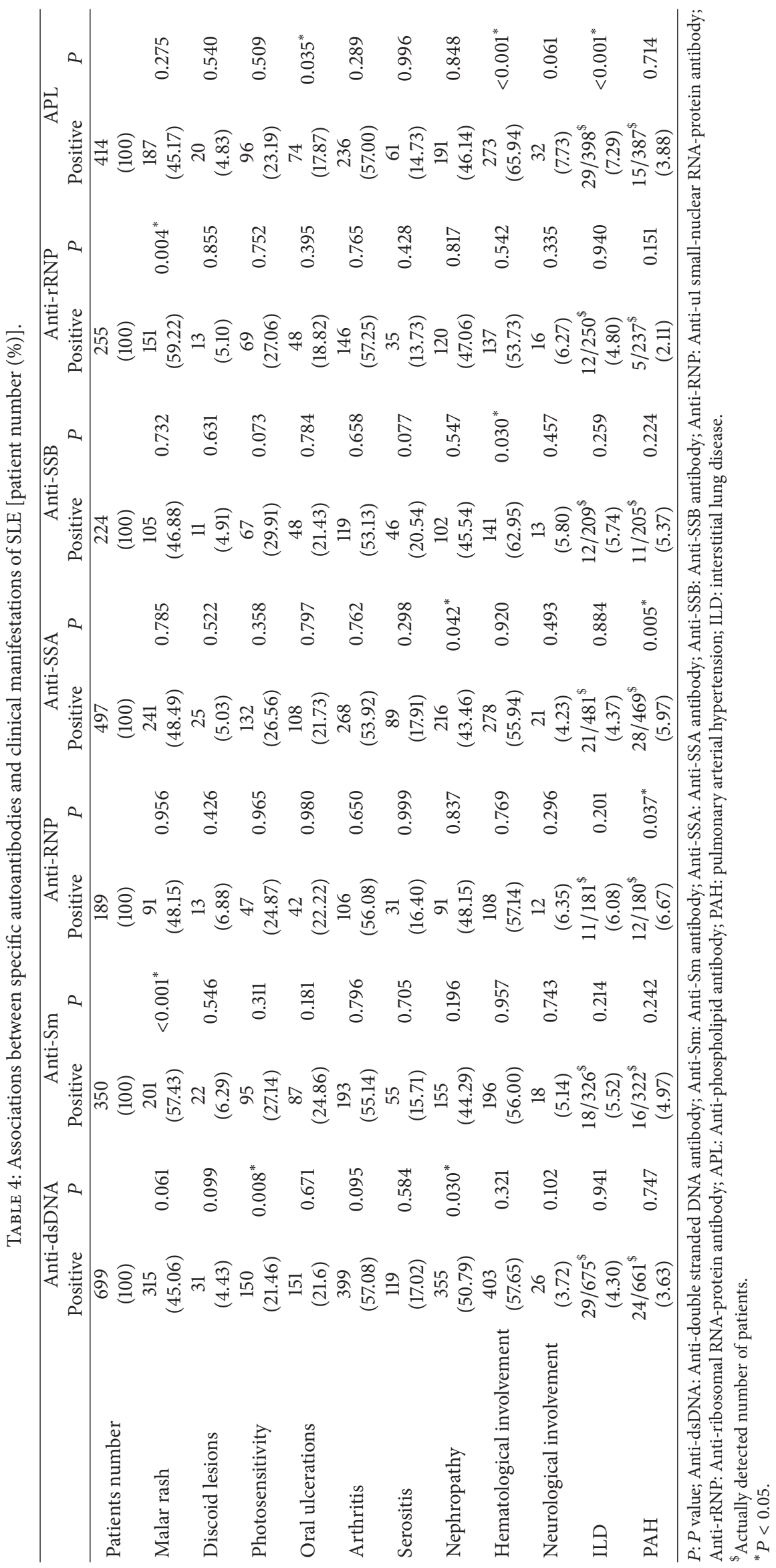




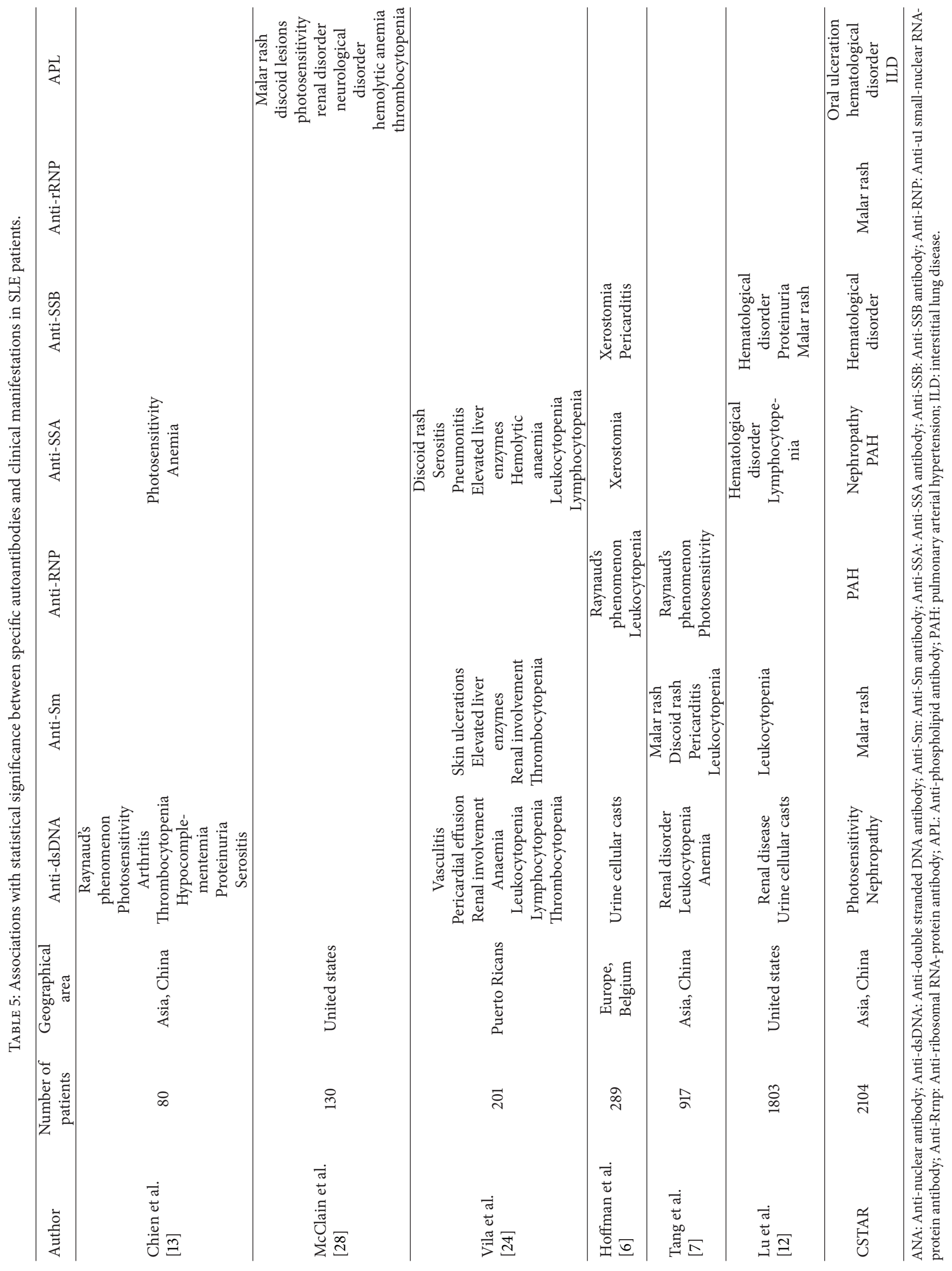




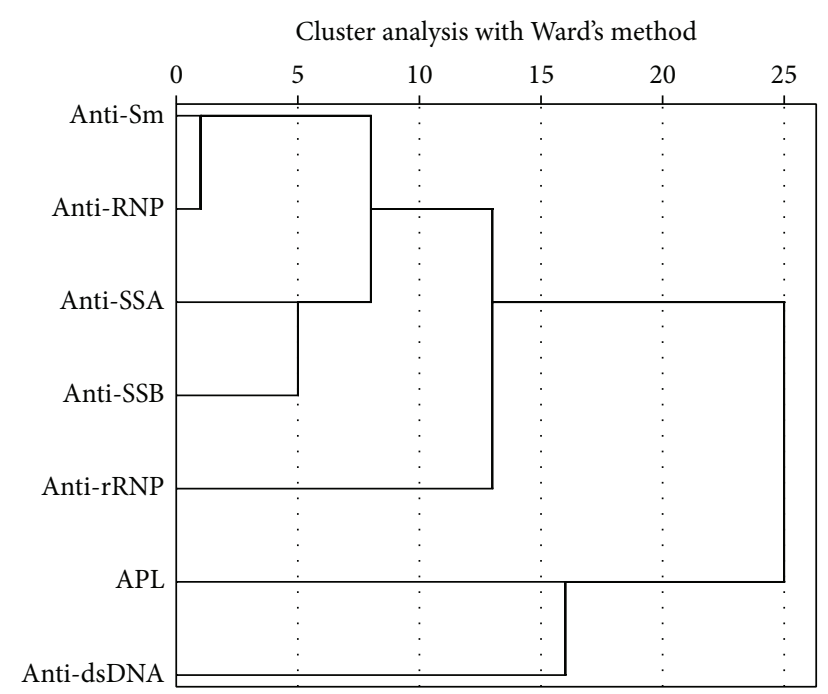

FIGURE 1: Result of cluster analysis with Ward's method in SLE patients. Five clusters of antibodies were identified. Cluster 1 consisted of antibodies to Sm and RNP and cluster 2 consisted of antibodies to SSA and SSB. Clusters 3, 4, and 5 consisted of antibodies to ribosomal $\mathrm{P}, \mathrm{dsDNA}$, and APL, respectively.

measurements due to its invasive characteristic but RHC is used for confirming the diagnosis of PAH according to the guideline. As far as we know, most of the 74 patients with a preliminary diagnosis of PAH identified in our study have been referred for confirmatory RHC, but these results were not recorded in the registry. This is a limitation of our study [34].

\section{Conclusion}

As the largest registry cohort study in China, CSTAR has already disclosed some clinical profiles of Chinese SLE patient $[8,34]$. Confirmation of the associations between clinical manifestations and specific autoantibodies found in our study can help physicians to understand the features of SLE patients better, especially in China. A thorough screening of ANA and anti-ENA antibodies when the diagnosis of SLE is established can help us to predict organ damage. We could focus on the specific autoantibody-related vital organ complications (e.g., PAH) in the follow-up of SLE patients. We suggest that repeating echocardiography annually may help to discover PAH at the early stage in SLE patients with anti-RNP and/or anti-SSA antibody. Early diagnosis of PAH in patients with SLE is important for initiating effective interventions to prevent malignant outcomes (e.g., heart failure).

\section{Abbreviations}

ANA: Anti-nuclear antibody

ENA: $\quad$ Extractable nuclear antigen

Anti-dsDNA: Anti-double stranded DNA antibody

Anti-Sm: Anti-Sm antibody
Anti-SSA: Anti-SSA antibody

Anti-SSB: Anti-SSB antibody

Anti-RNP: Anti-ul small-nuclear RNA-protein antibody

Anti-rRNP: Anti-ribosomal RNA-protein antibody

APL: $\quad$ Anti-phospholipid antibody

PAH: $\quad$ Pulmonary arterial hypertension

ILD: $\quad$ Interstitial lung disease.

\section{Conflict of Interests}

The authors declare that there is no conflict of interests regarding the publication of this paper.

\section{Authors' Contribution}

Jing Li, Xiaomei Leng, and Zhijun Li contributed equally to this study. All authors made substantial contributions to conception and design. Dr. Jing Li, Dr. Xiaomei Leng, and Dr. Zhijun Li performed the interpretation of data and wrote the paper. Dr. Jiuliang Zhao performed the analysis of data. Dr. Mengtao Li and Dr. Xin-Ping Tian revised the paper critically. Dr. Xiaofeng Zeng gave final approval of the version to be submitted. All authors read and approved the final paper.

\section{Acknowledgments}

Thanks are due to every rheumatologist working for CSTAR group in 104 centers for contribution in sample collecting. This study was supported by the Chinese National Key Technology R\&D Program, Ministry of Science and Technology (2008BAI59B02 and 2011BAI11B15), the Chinese National High Technology Research and Development Program, Ministry of Science and Technology (2012AA02A513), the Research Special Fund for Public Welfare Industry of Health, Ministry of Health (201202004), and National Major Scientific and Technological Special Project for "Significant New Drugs Development", Ministry of Science and Technology (2012ZX09303006-002).

\section{References}

[1] D. P. D'Cruz, M. A. Khamashta, and G. R. Hughes, "Systemic lupus erythematosus," The Lancet, vol. 369, no. 9561, pp. 587596, 2007.

[2] R. Cervera, M. A. Khamashta, J. Font et al., "Systemic lupus erythematosus: clinical and immunologic patterns of disease expression in a cohort of 1,000 patients. The European Working Party on Systemic Lupus Erythematosus," Medicine, vol. 72, no. 2, pp. 113-124, 1993.

[3] E. F. Borba, D. B. Araujo, E. Bonfa, and S. K. Shinjo, "Clinical and immunological features of 888 Brazilian systemic lupus patients from a monocentric cohort: comparison with other populations," Lupus, vol. 22, no. 7, pp. 744-749, 2013.

[4] M. F. Smikle, E. N. Barton, O. S. Morgan, and K. Deceulaer, "Photosensitivity and antinuclear antibodies in black patients with systemic lupus erythematosus," Journal of the Association for Academic Minority Physicians, vol. 7, no. 2, pp. 53-55, 1996. 
[5] M. R. Arbuckle, M. T. McClain, M. V. Rubertone et al., "Development of autoantibodies before the clinical onset of systemic lupus erythematosus," The New England Journal of Medicine, vol. 349, no. 16, pp. 1526-1533, 2003.

[6] I. E. Hoffman, I. Peene, L. Meheus et al., "Specific antinuclear antibodies are associated with clinical features in systemic lupus erythematosus," Annals of the Rheumatic Diseases, vol. 63, no. 9, pp. 1155-1158, 2004.

[7] X. Tang, Y. Huang, W. Deng, L. Tang, W. Weng, and X. Zhang, "Clinical and serologic correlations and autoantibody clusters in systemic lupus erythematosus: a retrospective review of 917 patients in South China," Medicine, vol. 89, no. 1, pp. 62-67, 2010.

[8] M. Li, W. Zhang, X. Leng et al., "Chinese SLE Treatment and Research group (CSTAR) registry: I. Major clinical characteristics of Chinese patients with systemic lupus erythematosus," Lupus, vol. 22, no. 11, pp. 1192-1199, 2013.

[9] M. C. Hochberg, "Updating the American College of Rheumatology revised criteria for the classification of systemic lupus erythematosus," Arthritis \& Rheumatism, vol. 40, no. 9, p. 1725, 1997.

[10] G. Ruiz-Irastorza, M. Garmendia, I. Villar, M. V. Egurbide, and C. Aguirre, "Pulmonary hypertension in systemic lupus erythematosus: prevalence, predictors and diagnostic strategy," Autoimmunity Reviews, vol. 12, no. 3, pp. 410-415, 2013.

[11] J. M. Cruse, R. E. Lewis, and S. Dilioglou, "Fate of immune complexes, glomerulonephritis, and cell-mediated vasculitis in lupus-prone MRL/Mp lpr/lpr mice," Experimental and Molecular Pathology, vol. 69, no. 3, pp. 211-222, 2000.

[12] R. Lu, J. M. Robertson, B. F. Bruner et al., "Multiple autoantibodies display association with lymphopenia, proteinuria, and cellular casts in a large, ethnically diverse SLE patient cohort," Autoimmune Diseases, vol. 2012, Article ID 819634, 2012.

[13] J.-W. Chien, C.-Y. Lin, and L.-Y. Yang, "Correlation between anti-Ro/La titers and clinical findings of patients with systematic lupus erythematosus," Zhonghua Yi Xue Za Zhi, vol. 64, no. 5, pp. 283-291, 2001.

[14] P. Alba, L. Bento, M. J. Cuadrado et al., "Anti-dsDNA, antiSm antibodies, and the lupus anticoagulant: significant factors associated with lupus nephritis," Annals of the Rheumatic Diseases, vol. 62, no. 6, pp. 556-560, 2003.

[15] J. D. Ni, X. Yao, H. F. Pan, X. P. Li, J. H. Xu, and D. Q. Ye, "Clinical and serological correlates of anti-Sm autoantibodies in Chinese patients with systemic lupus erythematosus: 1,584 cases," Rheumatology International, vol. 29, no. 11, pp. 1323-1326, 2009.

[16] S. C. Plastiras, S. P. Karadimitrakis, C. Kampolis, H. M. Moutsopoulos, and G. E. Tzelepis, "Determinants of pulmonary arterial hypertension in scleroderma," Seminars in Arthritis and Rheumatism, vol. 36, no. 6, pp. 392-396, 2007.

[17] N. Abid, A. S. Khan, and F. H. Otaibi, "Systemic lupus erythematosus (SLE) in the eastern region of Saudi Arabia. A comparative study," Lupus, vol. 22, no. 14, pp. 1529-1533, 2013.

[18] I. M. Gilboe, T. K. Kvien, T. Uhlig, and G. Husby, "Sicca symptoms and secondary Sjogren's syndrome in systemic lupus erythematosus: comparison with rheumatoid arthritis and correlation with disease variables," Annals of the Rheumatic Diseases, vol. 60, no. 12, pp. 1103-1109, 2001.

[19] B. R. Reed, L. A. Lee, C. Harmon et al., "Autoantibodies to SSA/Ro in infants with congenital heart block," The Journal of Pediatrics, vol. 103, no. 6, pp. 889-891, 1983.

[20] A. Menendez, J. Gomez, L. Caminal-Montero, J. B. DiazLopez, I. Cabezas-Rodriguez, and L. Mozo, "Common and specific associations of anti-SSA/Ro60 and anti-Ro52/TRIM21 antibodies in systemic lupus erythematosus," The Scientific World Journal, vol. 2013, Article ID 832789, 8 pages, 2013.

[21] S. C. Shiboski, C. H. Shiboski, L. Criswell et al., "American College of rheumatology classification criteria for Sjogren's syndrome: a data-driven, expert consensus approach in the Sjogren's International Collaborative Clinical Alliance cohort," Arthritis Care \& Research, vol. 64, no. 4, pp. 475-487, 2012.

[22] D. Launay, E. Hachulla, P.-Y. Hatron, X. Jais, G. Simonneau, and M. Humbert, "Pulmonary arterial hypertension: a rare complication of primary Sjogren syndrome: report of 9 new cases and review of the literature," Medicine, vol. 86, no. 5, pp. 299-315, 2007.

[23] F. J. Tapanes, M. Vasquez, R. Ramirez, C. Matheus, M. A. Rodriguez, and N. Bianco, "Cluster analysis of antinuclear autoantibodies in the prognosis of SLE nephropathy: are antiextractable nuclear antibodies protective?" Lupus, vol. 9, no. 6, pp. 437-444, 2000.

[24] L. M. Vila, M. J. Molina, A. M. Mayor, R. A. Peredo, M. L. Santaella, and S. Vila, "Clinical and prognostic value of autoantibodies in Puerto Ricans with systemic lupus erythematosus," Lupus, vol. 15, no. 12, pp. 892-898, 2006.

[25] I. Unal, C. Ceylan, F. Ozdemir, B. G. Turk, A. C. Kazandi, and G. Kandiloglu, "ITP as an initial manifestation of subacute cutaneous lupus erythematosus," The Journal of Dermatology, vol. 32, no. 9, pp. 727-730, 2005.

[26] M. L. Boey, C. L. Peebles, G. Tsay, P. H. Feng, and E. M. Tan, "Clinical and autoantibody correlations in Orientals with systemic lupus erythematosus," Annals of the Rheumatic Diseases, vol. 47, no. 11, pp. 918-923, 1988.

[27] E. Bonfa, S. J. Golombek, L. D. Kaufman et al., "Association between lupus psychosis and anti-ribosomal $\mathrm{P}$ protein antibodies," The New England Journal of Medicine, vol. 317, no. 5, pp. 265-271, 1987.

[28] M. T. McClain, M. R. Arbuckle, L. D. Heinlen et al., "The prevalence, onset, and clinical significance of antiphospholipid antibodies prior to diagnosis of systemic lupus erythematosus," Arthritis \& Rheumatism, vol. 50, no. 4, pp. 1226-1232, 2004.

[29] C. Sachse, K. Luthke, K. Hartung et al., "Significance of antibodies to cardiolipin in unselected patients with systemic lupus erythematosus: clinical and laboratory associations. The SLE Study Group," Rheumatology International, vol. 15, no. 1, pp. 23-29, 1995.

[30] M. A. Kanakis, V. Kapsimali, A. G. Vaiopoulos, G. A. Vaiopoulos, and M. Samarkos, "The lung in the spectrum of antiphospholipid syndrome," Clinical and Experimental Rheumatology, vol. 31, no. 3, pp. 452-457, 2013.

[31] A. P. van Dam, "Diagnosis and pathogenesis of CNS lupus," Rheumatology International, vol. 11, no. 1, pp. 1-11, 1991.

[32] D. L. Greenwood, V. M. Gitlits, F. Alderuccio, J. W. Sentry, and B.-H. Toh, "Autoantibodies in neuropsychiatric lupus," Autoimmunity, vol. 35, no. 2, pp. 79-86, 2002.

[33] P. G. Vlachoyiannopoulos, F. B. Karassa, K. X. Karakostas, A. A. Drosos, and H. M. Moutsopoulos, "Systemic lupus erythematosus in Greece. Clinical features, evolution and outcome: a descriptive analysis of 292 patients," Lupus, vol. 2, no. 5, pp. 303312, 1993

[34] M. Li, Q. Wang, J. Zhao et al., "Chinese SLE Treatment and Research group (CSTAR) registry: II. Prevalence and risk factors of pulmonary arterial hypertension in Chinese patients with systemic lupus erythematosus," Lupus, 2014. 


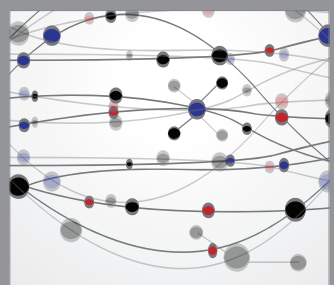

The Scientific World Journal
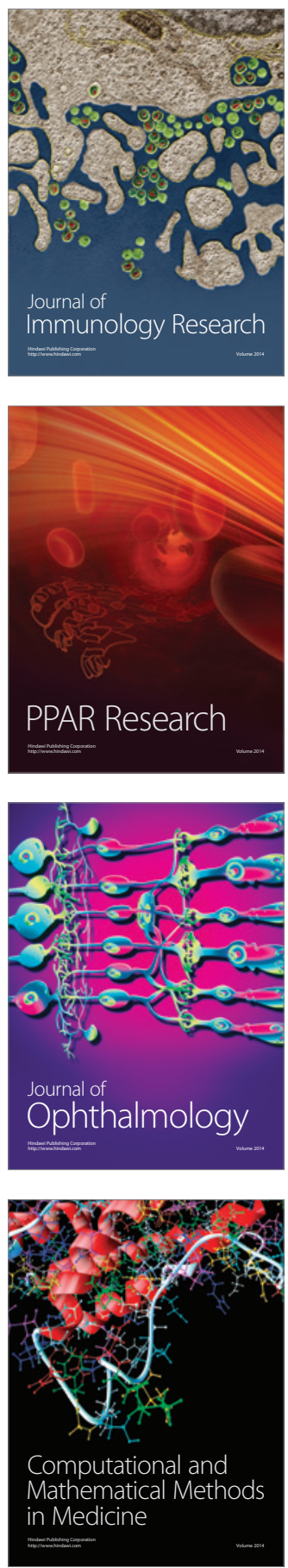

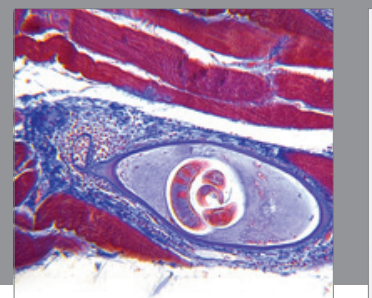

Gastroenterology

Research and Practice
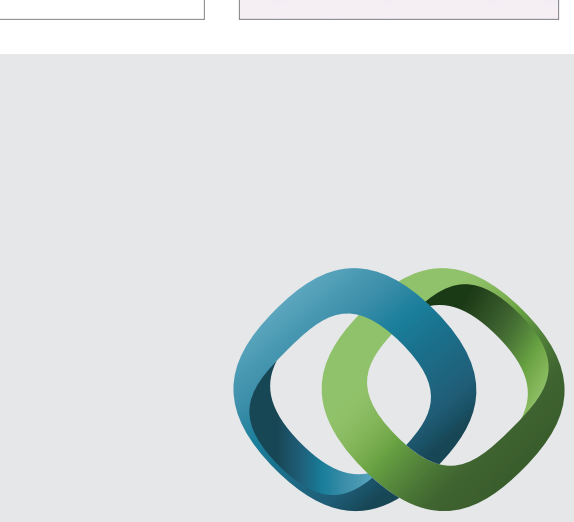

\section{Hindawi}

Submit your manuscripts at

http://www.hindawi.com
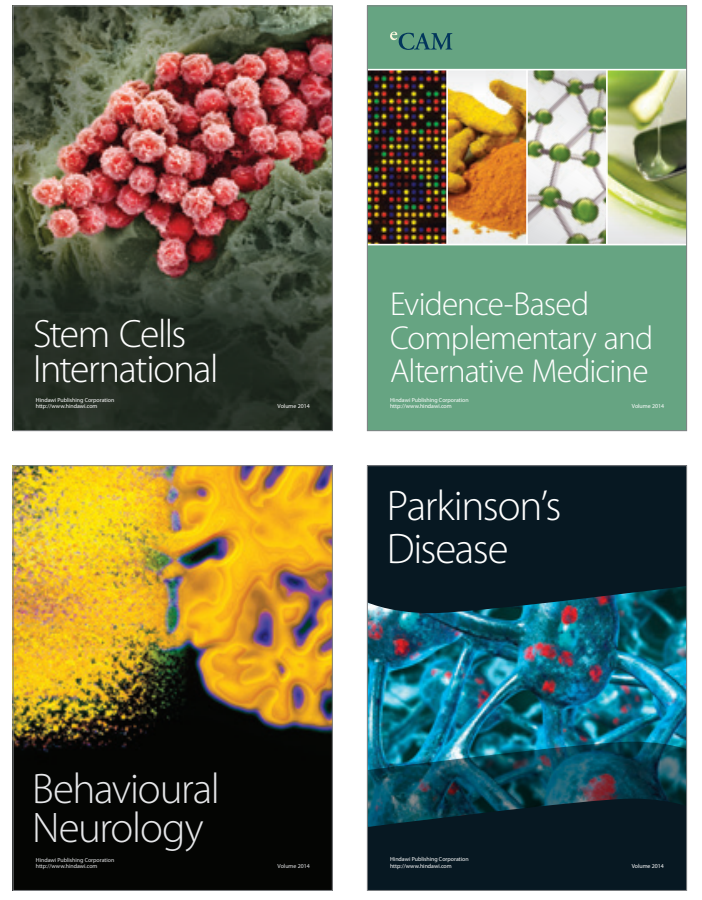
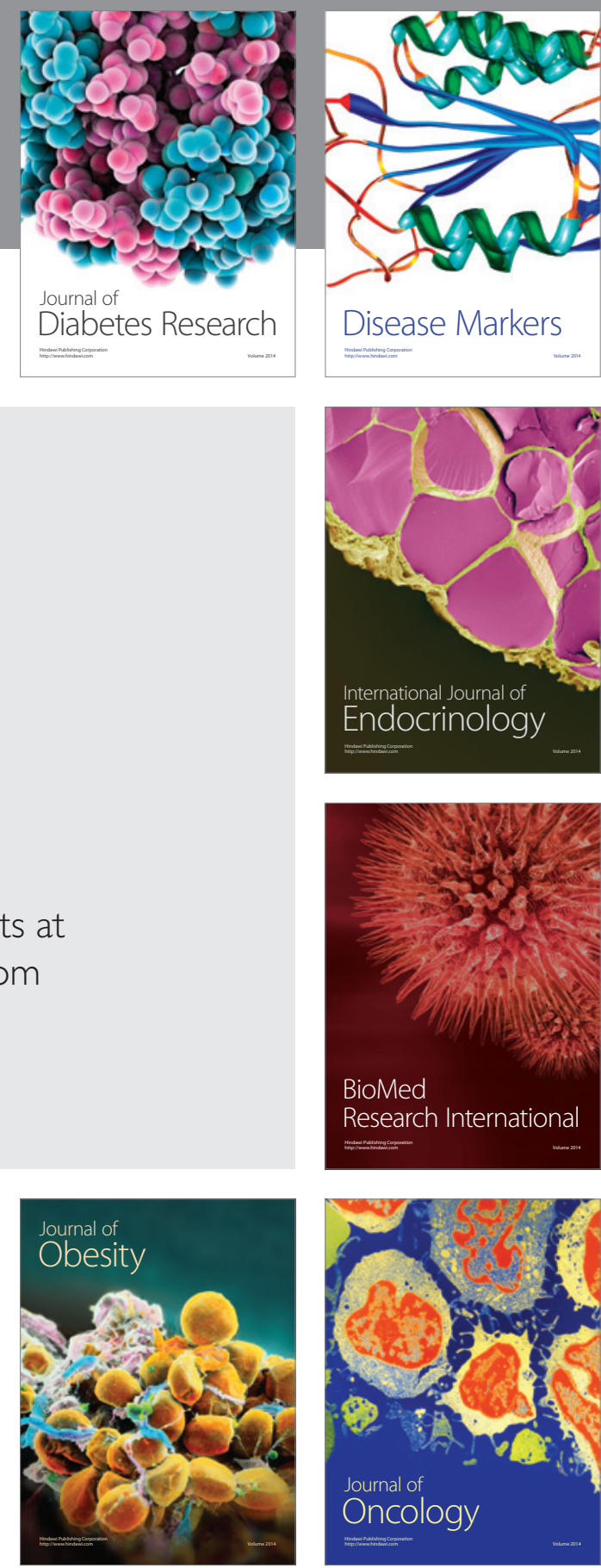

Disease Markers
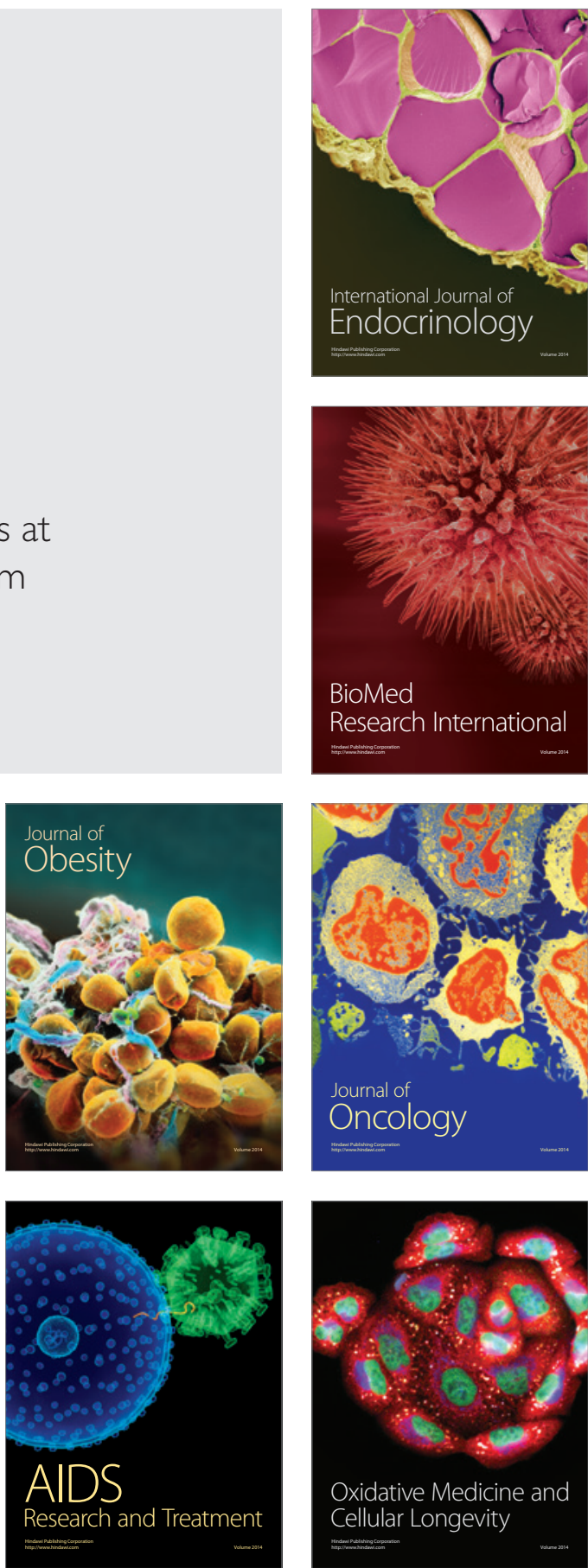